\title{
Die FMH intensiviert internationale Beziehungen
}

René Salzberg, während vieler Jahre ZV-Mitglied und "Aussenminister" der FMH, hat die Nachfolge von Alan Rowe als Generalsekretär der EFMA angetreten. Die EFMA, das European Forum of Medical Associations, ist ein Diskussionsforum für die WHORegion "Europa" und für die nationalen Ärztegesellschaften der 51 Staaten, welche diese Region umfasst. Wie bekannt kämpfen die WHO und damit auch die EFMA mit grossen Finanzproblemen. Obwohl die WHO der EFMA für die nächsten drei Jahre eine Unterstützung von jährlich 50000 US-Dollar zugesprochen hat, bleibt eine Finanzierungslücke von 15000 bis 20000 US-Dollar für die Unterstützung der finanzschwachen Staaten der ehemaligen Sowjetunion bestehen. Der ZV der FMH hat beschlossen, Kosten der EFMA im Sinn des Naturalleistungsprinzips zu übernehmen und Sekretariatsdienstleistungen für den Generalsekretär à fonds perdu anzubieten. Die SÄZ wollte vom FMH-Präsidenten, Dr. Hans Heinrich Brunner, und von Dr. René Salzberg wissen, welche Gründe für dieses internationale Engagement gesprochen haben.

SÄZ: Was hat den Zentralvorstand zu dieser recht grosszügigen Geste bewegt, wo doch die Kriegskasse der FMH nicht gerade voll ist?

H. H. Brunner: Das Engagement hält sich doch in Grenzen. Es sind ja nicht Tausende von Franken, die in diese Sache fliessen. Immerhin führt dieses Engagement zu einer zusätzlichen Belastung des Vorstandssekretariats, welche Sekretariatsdienstleistungen übernehmen wird. Und das ist angesichts der jetzt schon massiven Belastung ein nennenswerter Beitrag an die EFMA.

\section{Ist diese Unterstützung nicht nur ein symbolischer} Akt?

Absolut nicht. Wir sind der Auffassung, dass es die EFMA wirklich braucht. Es braucht sie als ein Ort der Diskussion und der Positionsfassung in einem sich integrierenden Europa. Es braucht sie auch, um die Staaten der ehemaligen Sowjetunion bzw. Staaten des ehemaligen Warschauer Paktes einbeziehen zu können. Was dort gesundheitspolitisch geschieht oder eben nicht geschieht -, hat eminente Auswirkungen auf alle westeuropäischen Länder und damit auch auf die Schweiz. Denken wir zum Beispiel an die Tuberkuloseepidemie oder an die venerischen Erkrankungen. Es gibt andere Gremien wie das «Comité permanent" ${ }^{1}$, das im Banne der EU steht. Zu diesem manchmal sehr bürokratisch daherkommende EuroZug stellt die EFMA für Nicht-EU-Staaten wie die Schweiz und Länder der östlichen Sphäre ein Gegengewicht dar. René Salzberg hat während seiner 17jährigen Tätigkeit für die FMH in einer Art Funktion des Aussenministers unschätzbare Aufbauarbeit

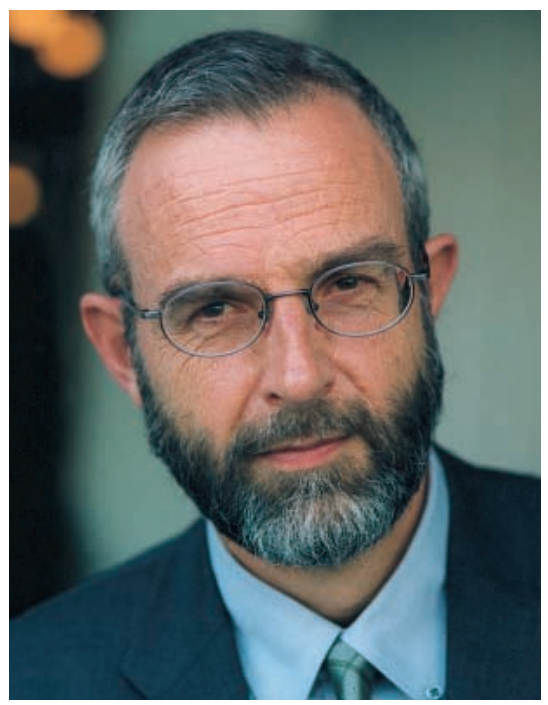

Dr. Hans Heinrich Brunner

innerhalb dieses Gremiums geleistet. Wir sind froh, dass er diese Arbeit auch weiterhin leisten kann. Aber das Aussenministerium muss jetzt durch den Präsidenten, der wirklich die gute Hoffnung hat, endlich vom TARMED entlastet zu werden, wahrgenommen werden. Die Schweiz war bisher durch die FMH oder durch den Zentralvorstand in Europa zu wenig präsent. Diese Absenz wird oft kritisiert und bedauert, weil die Mitarbeit der Schweiz an sich sehr gefragt wäre: Wir gelten als relativ neutral, unbestechlich und effizient. Mit unserem Engagement haben wir nun auch die Möglichkeit, das Image der Schweiz zu verbessern.

Ist dies mitunter auch die Gelegenheit, Dinge nachzuholen, die man während der geradezu besessenen Beschäftigung mit TARMED versäumt hat?

Die Versäumnisse haben damit angefangen, dass die FMH - nicht anders als die Schweiz - sich als eine Insel der Seligen verstanden hat. Das hat sich grundsätzlich geändert, und wir haben gelernt, dass auch die Ärzteschaft in der Schweiz nicht mehr eine Insel der Seligen ist. Es ist eine Realität, dass sich unsere Grenzen auch für Ärzte öffnen. Zweifelsohne haben wir uns die letzten vier bis fünf Jahre viel zu stark mit TARMED-Fragen beschäftigt und haben Entwicklungen übersehen, die über das Schicksal der Ärzteschaft letztlich viel wichtiger sein werden als das Tarifwerk.

1 Das "Comité permanent» ist eine Institution der Europäischen Union, welche Ärzte aus allen EU-Ländern und aus alle medizinischen Disziplinen vereint und dem Europäischen Rat für medizinische Fragen beratend zur Verfügung steht 
Was sind die Stärken der EFMA?

Die WHO ist eine staatliche konzipierte und von nationalen Regierungen finanzierte Organisation, die aber begriffen hat, dass eine rein bürokratische, am grünen Tisch ausgedachte Gesundheitspolitik zu wenig konkreten Resultaten führen würde und dass Ärzte daran beteiligt werden müssen. Aus diesen Überlegungen heraus ist dann im Schosse der WHO die EFMA geschaffen worden, um mit den Ärzten zu kooperieren und deren Know-how nutzbar zu machen. Dass dabei ein gewisser Antagonismus zwischen den beiden Denkrichtungen zutage tritt und dass Ärztegesellschaften mitteleuropäischen Raumes, die noch das Prinzip der Selbstverwaltung kennen, gelegentlich in Oppositionshaltung gehen, ist fast natürlich und erweist sich letztlich nicht als kontraproduktiv, ja ist im Grunde recht fruchtbar.

Die WHO kämpft mit Finanzproblemen. Ist diese Organisation überhaupt zu irgendwelchen signifikanten Handlungen fähig?

Sie ist sicher handlungsfähig. Man könnte sich aber fragen, ob die WHO in all ihren Tätigkeiten richtig und zweckmässig gehandelt hat und handelt. Darüber kann man sicher verschiedener Meinung sein. Aber finanziell ist die WHO handlungsfähig.

Trotzdem haben gerade die Staaten des ehemaligen Warschauer Paktes grosse Mühe, ihre Beiträge zu zahlen.

Gerade deshalb hat auch schon der Vorgänger des jetzigen Generalsekretärs, Dr. Alan Rowe, versucht, den Kontakt mit diesen Staaten zu knüpfen und sie in Europa zu integrieren. Dieses Ziel ist richtig und Fakt ist, dass diese Staaten völlig verarmt sind und dass ihre Ärztegesellschaften über kein Geld verfügen. Hier liegt ein ganz konkreter Ansatz für zukünftige Aktivitäten der FMH. Wir möchten mit drei, vier Ärztegesellschaften in diesem Raum konkret zusam-

\section{The aims of the EFMA}

The aims of the European Forum of Medical Associations and WHO are to establish dialogue and cooperation between National Medical Associations (NMAs) and WHO in the European Region to improve the quality of health and health care in Europe; promote the exchange of information and ideas between NMAs and WHO; integrate appropriate aspects of policies for health for all into basic, postgraduate and continuing medical education; and formulate consensus policy statements on health issues.

http://www.who.dk/ch/cor/EFMAintro.htm

\section{WHO-Regions}

Africa

Americas/Pan American Health Organization

Eastern Mediterranean

Europe

South-East Asia

Western Pacific menarbeiten und ihnen zeigen, wie eine nach dem Selbstwartungsprinzip organisierte Ärzteschaft relativ billig funktionieren kann. Diese wiederum haben grosses Interesse signalisiert und Einladungen sind gefolgt. Das ist in meinen Augen eine vernünftige Unterstützung, sie beruht auf dem Prinzip ärztlicher Kollegialität und trägt auch langfristig zu unserem guten Image bei.

\section{Es geht also um Entwicklungshilfe!}

Ja, um Entwicklungshilfe in ihrer fast konstitutionellen Ambivalenz: Sie wird von moralischen Prinzipien getragen, ist aber doch nie frei von potentiellem materiellem Gewinn.

Hatten Sie schon Kontakte mit einzelnen Ärztegesellschaften aus Osteuropa?

Wir haben diese Kontakte und sind bereits Einladungen in die Ukraine und nach Weissrussland gefolgt.

\section{Bewaffnet?}

In diesen Staaten ist man als Gast relativ sicher. Manchmal habe ich den Eindruck, ich müsste mich eher hier in der Schweiz bewaffnen, etwa wenn es um TARMED geht.

Aber Sie befürworten bewaffnete Auslandseinsätze von Schweizer Ärzten?

Ich würde Auslandseinsätze von Schweizer Ärzten, deren Selbstschutz gewährleistet ist, jederzeit befürworten. Damit sehen Sie, dass ich meiner politischen Neutralität verpflichtet bin und mich keinem politischen Lager zuordnen lasse (Auftrag der Ärztekammer erfüllt).

In welche Länder beabsichtigen Sie noch zu reisen? Es wären Reisen nach Kirgistan und Kasachstan angesagt gewesen, aber TARMED-Termine haben das verhindert.

Entwicklungshilfe hat ja immer auch ein bisschen einen missionarischen Aspekt. Geht es darum, den Russen zu zeigen, wie man Weiterbildung betreibt, wie man Facharzttitel verleiht, ja sogar, wie man einen Tarif einführt?

Viele dieser Ärztegesellschaften fühlen sich durch gewisse europäische Staaten majorisiert und versuchen, eine Gegenposition einzunehmen. Solche Bestrebungen kann die FMH unterstützen. Die FMH hat ja grosse Erfahrungen im Umgang mit Minoritäten und widerlaufenden Tendenzen: Wer TARMED durchgestanden hat, kann auch in Kasachstan eine selbstlose Hilfe leisten.

Sie sprechen sicher auch die in diesem Zusammenhang gemachten Erfahrungen mit unseren Apparatschiks an?

Mit internen und externen Apparatschiks, aber vor allem mit den externen, die es in diesen Ländern sicher auch gibt. Anderseits wünscht man sich in diesen Staaten wieder etwas mehr funktionierende bürokratische Strukturen. Wenn ich an das Ausmass an 
fehlenden Strukturen und an die Gesetzlosigkeit in diesen Ländern denke, weiss ich manchmal das überdeterminierte bürokratische System unseres Landes wieder etwas zu schätzen.

Wo besteht nun ganz konkret am dringendsten Handlungsbedarf in diesen Ländern? Was wären erste Hilfemassnahmen, die man sich vorstellen könnte? Oder was erwartet man von der FMH? Die Ärztegesellschaften erwarten, dass man ihnen hilft, ihre Organisation aufzubauen, damit sie selbständig funktionieren können. Das kann damit beginnen, dass man zeigt, wie man das Sekretariat einer Ärztegesellschaft organisieren kann oder wie man einen PC benützt. Die bestehenden Ärztegesellschaften sind wenig strukturiert. Zum Teil sind die Verhältnisse nicht durchsichtig und wir wissen nicht immer mit Bestimmtheit, mit welchen Interessengruppen unsere Ansprechpartner assoziiert sind. Wir müssen uns immer vergewissern, dass wir unsere Hilfe dort anbieten, wo die Basisärzteschaft wirklich dahintersteht. Die meisten Gesellschaften sind sehr arm, es fehlt ihnen an der bescheidensten Infrastruktur, das Geld verschwindet über dubiose Kanäle - so wie wir das aus dem gesamtpolitischen Bereich bereits kennen. Die Leute wünschen sich aber wirklich, daraus herauszukommen. Es ist immer auch beeindruckend und stimmt nachdenklich, wenn man sieht, wie medizinisch hochqualifizierte Ärzte unter unsäglichen ökonomischen Randbedingungen in einer fast entwürdigenden Art und Weise arbeiten müssen. Hinzu kommt, dass sie seit 70 oder mehr Jahren von irgendwelchen regelmässigen Kontakten mit dem Rest der Welt abgeschnitten waren und das jetzt nachholen müssen. Auch hier können wir grosse Hilfe leisten. $\mathrm{Zu}$ erwähnen ist auch der Aspekt der Aus-, Weiter- und Fortbildung, der für diese Länder sehr wichtig ist. Auch hier können wir etwas bieten, damit hat sich gerade René Salzberg seit Jahrzehnten beschäftigt. Wir werden unsere Hilfe über Austauschprogramme anbieten, aber auch die Möglichkeiten der modernen Kommunikationstechniken nutzen.

Ist auch eine Unterstützung im medizinischen Bereich vorgesehen, oder betrifft sie nur organisatorische Aspekte?

Die FMH kann keine direkte Hilfe im medizinischen Bereich anbieten, aber sie wird sicher ihre Dienste anbieten, etwa wenn es darum geht, die Zweckmässigkeit medizinischer Entwicklungshilfe mitzubeurteilen. Es besteht immer die Gefahr, dass man den Leuten Dinge hinstellt, die sie in ihren Versorgungsstrukturen gar nicht brauchen können.

Diese Aktivitäten öffnen auch das Tor zu einem gewissen Tourismus unter dem Deckmantel der Entwicklungshilfe. Man wird in diesen Gegenden viel herumreisen und sich freundliche Worte spenden, vielleicht ein bisschen Hilfe.

Ist das nicht ein Tropfen auf einen heissen Stein? Die FMH will überhaupt nichts von Ärztetourismus wissen. Wir werden zielgerichtet und punktuell Leute einladen, und wenn wir eigene Leute in diese Länder schicken, dann haben sie dort einen Job zu erledigen. Es liegt mir persönlich sehr am Herzen, jegliche touristischen Elemente von diesen standespolitischen Aktivitäten fernzuhalten. Ich habe mir diesbezüglich einen nicht immer beliebten Namen eines absolut konsequenten Jüngers von Calvin eingehandelt. Aber ich würde es nie dulden, dass gerade in diesem Bereich luxuriöse Elemente aufkommen würden: etwas anderes als Economy-Flüge und durchschnittliche Hotels kommen nicht in Frage.

\section{Besteht zu den Gremien der Europäischen Union} nicht eine gewisse Konkurrenzsituation?

Latent ist diese Konkurrenzsituation sicher gegeben. Das von gewissen Ärztegesellschaften der EU zum Ausdruck gebrachte Selbstverständnis - wenn es z.B. um wichtige Entscheidungen geht - stösst bei den Oststaaten nicht immer auf Gegenliebe. Manchmal spielen da auch ungute Reminiszenzen aus vergangenen Zeiten eine nicht zu unterschätzende Rolle. Solche Sensibilitäten müssen unbedingt berücksichtigt werden. Wir sehen unsere Aufgabe auch darin, für einen Ausgleich zu sorgen. Wir hoffen, beim "Comité permanent" mit solchen Anliegen gehört zu werden und auf diese Entwicklungen hinweisen $\mathrm{zu}$ können. Die von der Schweiz als Kooperation und nicht als passives Verharren gelebte Neutralität kann da schon sehr positive und beispielhafte Wirkung entfalten.

Aussenpolitik hat auch immer die Aufgabe, die Türen für ökonomische Beziehungen zu öffnen. In den Oststaaten können bekanntlich klinische Studien sehr billig, rasch und in grossem Massstab durchgeführt werden. Ist es nun Ihre Rolle, unserer Pharmaindustrie den Gesundheitsmarkt Osteuropa $z u$ erschliessen?

Die FMH hat mit diesen wirtschaftlichen Interessen in der Schweiz nichts am Hut und erweist sich als weitgehend resistent gegenüber Druckversuchungen und Liebesbezeugungen interessierter Kreise. Wenn solche wirtschaftlich prosperierende Zusammenarbeiten entstehen sollen, dann kann das nach unserer Auffassung nur in einem Klima des gegenseitigen Respekts und des gemeinsamen Nutzens geschehen. Nach meinen Kenntnissen aber sind solche wirtschaftlichen Projekte häufig nur Tagesblüten und werden von diesen Staaten zunehmend als ausserordentlich negativ wahrgenommen. Diese Staaten spüren, dass wir eine Zusammenarbeit anstreben, die wohl auch etwas gemeinsames Wirtschaftliches nicht ausschliesst, aber vor allem zum Ziel hat, eine nachhaltige Wirkung zu entfalten. Das wird positiv wahrgenommen. Allerdings werden weite Kreise der Oberschicht in diesen Ländern von einer hemmungslosen Geldgier getrieben und da gibt es natürlich auch Ärztekreise, die solchen Versuchungen erliegen. Ich möchte nochmals betonen: die FMH ist weder abhängig noch Mittlerin solcher wirtschaftlichen Interessen. Wir sind in diesem Sinne eine völlig unabhängige und auch neutrale berufliche Organisation. 
Wer kann an diesen FMH-Projekten mitarbeiten? Könnten jüngere Kollegen hier einen Weg finden, ihren Horizont zu öffnen?

Es scheint mir durchaus möglich, zum Beispiel Austauschprogramme für Kollegen in der Weiterbildung mit interessierten Spitälern zusammen zu erarbeiten. Ich kann mir aber auch vorstellen, dass gestandene Standespolitiker Leute empfangen, oder dass sie vor Ort gewisse Ratschläge geben, wenn das erwünscht ist. Das würde ich sehr von den momentanen Gegebenheiten abhängig machen.

Herr Dr. Salzberg, Sie sind zum neuen Generalsekretär der EFMA gewählt worden. Worin liegen die ersten Herausforderungen in dieser Funktion? René Salzberg: Wenn nun die FMH mit meiner Person und das Vorstandssekretariat mit Frau Rüegg die Sekretariatsdienstleistung der EFMA übernehmen, so fällt zunächst im Vergleich mit meinem Vorgänger, Dr. Alan Rowe von der British Medical Association auf, dass wir das Handicap des viel kleineren Beziehungsnetzes haben. Es ist deshalb eine meiner ersten Aufgaben, diese persönlichen Beziehungen aufzubauen. Dann muss ich die prioritären Aufgaben für die nächsten drei Jahre definieren und feststellen, welche Probleme dringend angepackt werden müssen.

\section{Woran denken Sie?}

Ein Thema ist die Globalisierung mit ihren Chancen und Gefahren für die Ärzteschaft. Das Forum ist sich bewusst, dass die gegenwärtigen Diskussionen der WTO (World Trade Organisation; Anm. der Red.) sehr wohl auch auf die Gesundheitsversorgung verschiedener Länder einen Einfluss haben können und hat eine entsprechende Position verfasst. ${ }^{2}$ Mit Sicherheit werden uns die Fragen beschäftigen, welche sich mit der Aufschlüsselung des menschlichen Genoms stellen, wie etwa das kontrovers diskutierte Problem der Patentierung oder die Aufschlüsselung des Genoms der Bevölkerungen von Island und Lettland. Dann sind auch die Menschenrechte in verschiedenen Ländern der EFMA ein Thema von grosser Aktualität. Die ärztliche Aus-, Weiter- und Fortbildung beschäftigt uns, weil viele Länder diese Probleme sehr unstrukturiert angehen. In anderen Ländern aber, wie beispielsweise Grossbritannien oder Deutschland, entwerfen die Regierungen Gesetzesinitiativen, welche die Ärzte viel stärker verpflichten sollen, ihre Fortbildung nachzuweisen und zu zeigen, welches die nützlichen und welches die nicht nützlichen Formen der ärztlichen Fortbildung sind.

Wie wird Ihre Arbeit konkret aussehen?

Es gibt da einiges zu tun: Ich werde mich um organisatorische und finanzielle Fragen kümmern müssen. Das Forum hat kein Geld, weil es keine gesetzlichen Strukturen hat. Es ist eine Arbeitsgemeinschaft, die sich einmal im Jahr in einem Land während zwei bis drei Tagen trifft. Die Teilnehmer bezahlen eine Teilnahmegebühr, die bisher vom Organisator auch gebraucht wurde. Ich stelle mir vor, dass man in $\mathrm{Zu}$ kunft daraus ein bisschen Geld für die Infrastruktur

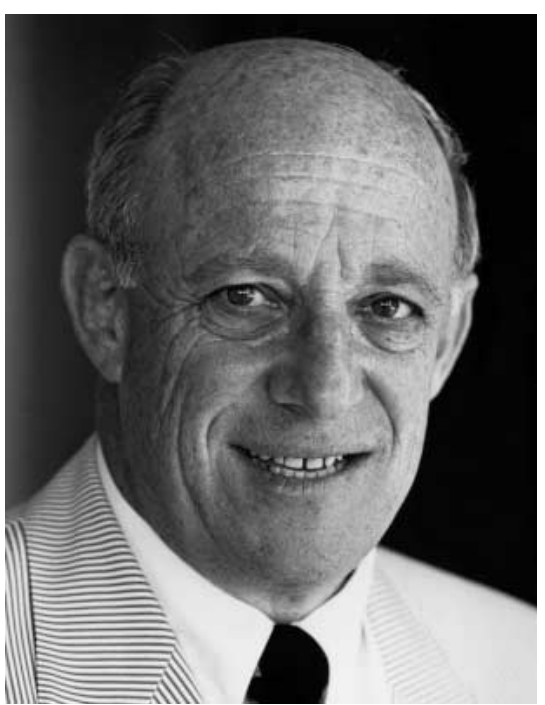

Dr. René Salzberg

des Forums ableiten könnte. All das diskutieren wir jetzt innerhalb der FMH und zusammen mit den anderen Mitgliedstaaten des Forums. Es ist «work in progress" in infrastruktureller, organisatorischer aber auch programmatischer Beziehung. Natürlich ist der Generalsekretär nicht der "gentil organisateur" oder der "maître de plaisir». In solch einer Organisation braucht es den Input aller Länder und der WHO. Es müssen Themen vorgeschlagen werden ebenso wie geeignete Personen, welche diese darstellen können.

Sie sind ja auch noch Vizepräsident bei der UEMS ${ }^{3}$ und können sicher beurteilen, ob sich die verschiedenen supranationalen Organisationen in ihren Aufgabengebieten überschneiden. Finden da nicht redundante Aktivitäten statt, die man eigentlich bei knappen Ressourcen bündeln sollte?

Diese Frage ist sehr berechtigt. Sie stellt sich bereits auf nationaler Stufe. Aus meiner Tätigkeit in der Schweiz kenne ich das Phänomen, die gleichen Persönlichkeiten in verschiedenen Gremien zu ähnlichen Themen immer wieder zu treffen. Das ist im Aus-, Weiter- und Fortbildungssektor sehr ausgeprägt der Fall und es ist natürlich naheliegend, dass dies auch im internationalen Bereich geschieht. Das «Comité permanent des médecins européens», die Organisa-

2 "Whilst consensus could not be achieved on a formal position, the Forum agreed that National Medical Associations should take note of the possible developments and approach their governments to ensure that in any World Trade Agreement negotiations the principles of equity and solidarity in the provisions for health are for all citizens should not be in anyway eroded by deregulation or other measures to promote trade." EFMA, Ljubljana, Meeting of the Forum, 9-11 March 2001.

3 UEMS ist die Union Européenne des Médecins Spécialistes, eine Organisation der EU, welche Vertreter der wichtigsten medizinischen Fachdisziplinen umfasst. Die Website befindet sich unter www.uems.org. Vgl. auch das Interview mit Dr. R. Salzberg: Europa - auch eine Chance für die Schweizer Ärztinnen und Ärzte? Schweiz Ärztezeitung 2000;81(1):9-13. 
tionsstruktur der EU-Ärztegesellschaften, der die Schweiz nur als Beobachter angehört, bemüht sich zur Zeit, genau diese Thematik anzugehen: Doppelspurigkeiten sollen vermieden werden und die verschiedenen Organisationen sollen nur das machen, wozu sie eigentlich geschaffen wurden.

\section{Mit anderen Worten, eine Beschränkung auf das Kerngeschäft zu fördern?}

Die meisten supranationalen Organisationen in $\mathrm{Eu}-$ ropa, die Sie angesprochen haben, also UEMS, UEMO, AEMH sind Ärzteorganisationen der Staaten der Europäischen Union. Nach und nach kommen noch andere Länder als Beobachter dazu und in ein paar Jahren wird sich die EU noch erweitern. Nun darf man aber nicht vergessen, dass die WHO-Region "Europa" 51 Staaten umfasst. Es ist ein langer Weg, bis alle diese in einer Europäischen Union vereinigt sein werden. Es gibt zwar Fragen, welche im "Comité permanent" und in der UEMS besprochen werden, die aber in keiner Art und Weise verbindlich oder überhaupt nur bekannt sind in Ländern, die nicht diesen Organisationen angehören. Es besteht daher ein grosser Bedarf an Information und Diskussion. Es ist die Einzigartigkeit des Forums, dass es alle Länder umfasst, EU- und Nicht-EU-Länder.

In diesem Sinne richtet sich ein Hauptinteresse des Forums auf die Länder der ehemaligen Sowjetunion. Kennen Sie diese Länder, etwa aus persönlichen Beziehungen, und wissen Sie, was diese Länder von der EFMA erwarten?

Es gibt dort grosse Unterschiede. Es gibt Länder, denen es besser, und Länder, denen es weniger gut geht; in entsprechender Verfassung befinden sich die jeweiligen Ärzteverbände. Es gibt Länder, in welchen es schon vor der kommunistischen Ära Ärzteorganisationen gegeben hat, die heute zu neuem Leben erwachen und auf eine gewisse Tradition zurückgreifen können. Das sind vor allem die Länder Zentraleuropas: Ungarn, Polen, die Tschechische Republik. Wir haben einen guten Kontakt zu den dortigen Ärzteorganisationen, die bereits in den vergangenen Jahren an den Forumsveranstaltungen teilgenommen haben, gut strukturiert sind und die Bedingungen für nationale Ärztegesellschaften durchaus erfüllen. Dann gibt es Länder, in welchen Ärztevereinigungen in der Form, wie wir sie kennen - als freie, demokratisch gewählte und unabhängige Ärzteorganisationen -, nicht existieren, und wo die Ärzte früher nur als verlängertes Organ des Gesundheitsministeriums funktioniert haben.

\section{Wie kann man gerade diesen benachteiligten Ländern helfen?}

In Ländern der ehemaligen Sowjetunion wie Kasachstan, Kirgistan und Georgien bestehen Ärzteverbände, bei denen wir nicht immer ganz sicher sind, ob sie den gewünschten Standards wirklich entsprechen. Immerhin strecken diese Verbände ihre Fühler aus und interessieren sich für unsere westlichen Organisationsformen. Wir hoffen deshalb, dass Vertreter dieser «Stan-Staaten» nächstes Jahr zur nächsten
Versammlung nach Wien kommen können. Dafür wollen wir auch die nötigen finanziellen Mittel bereitstellen. Sie sollen kommen, hören und Informationen mitnehmen können. Ich sehe allerdings eine Gefahr darin, dass gewisse nun meinen, das allein selig machende Konzept zu haben und dass eine Art Kolonialismus entsteht. Ich vertrete klar die Meinung, dass wir unser System nicht tel quel exportieren sollten. Wir müssen die Information zur Verfügung stellen und vorerst nur erläutern, wie wir gewisse Dinge anpacken; es bleibt dann in der Verantwortung der einzelnen Länder, das daraus zu entnehmen, was sie wirklich brauchen. Gerade an diesem sensiblen Punkt hat die Schweiz die vornehme Rolle des Mediators zwischen hochindustrialisierten Gebieten und den Gebieten in Entwicklung wahrzunehmen.

\section{Was würden Sie diesen Organisationen bei der} Aufbauarbeit empfehlen?

Es gibt eine ganze Kaskade von möglichen Empfehlungen. Zuerst muss ein Arzt als Arzt überleben können. Er muss ein Einkommen haben. Er muss eine Position haben, die es ihm erlaubt, seinen Arztberuf medizinisch unabhängig in einem demokratisch akzeptierten Gesundheitssystem auszuüben. $\mathrm{Ob}$ das nun in einem nationalen Gesundheitssystem oder in einem System ist, wie wir es in der Schweiz kennen, ist unwichtig. Wichtig ist, dass er leben kann. Das zweite ist, dass er eine gute, im ökonomischen Rahmen seines Landes mögliche Medizin praktiziert. Wir müssen auch in erzieherischem Sinne wirken und die Aus-, Weiter- und Fortbildungsstandards kommunizieren, die uns richtig erscheinen. Wir müssen die gesundheitsökonomischen Diskussionen führen, die auch in unserem Lande je länger je wichtiger werden. Last but not least sind die eingangs erwähnten Menschenrechte und die Fragen der ärztlichen Ethik ausserordentlich wichtig, gerade in Ländern, die während Jahrzehnten diese Aspekte vernachlässigt haben.

Sind diese Länder, die jetzt Aufbauarbeit benötigen, nicht gefährdet, dass sie eingenommen werden als Versuchsfelder?

Das ist eine grosse Gefahr und ich kann Ihnen dazu ein konkretes Beispiel nennen. Die Ärztevereinigung von Kasachstan hat vor drei Jahren den Führungsausschuss, das sogenannte Liaisonkomitee des Forums, zu einer Sitzung nach Almati eingeladen, und der Grossteil der Mitglieder des Liaisonkomitees ist dann tatsächlich auch nach Almati geflogen. Als sie dort ankamen, mussten sie feststellen, dass die ganze Sache von einer grossen deutschen Medizinapparatefirma und von einer Vereinigung von grossen Privatspitälern gesponsert war. Gefahren verschiedenartiger Vereinnahmungen bestehen also in der Tat, und wir versuchen natürlich, dem entgegenzuwirken. Anderseits kann die WHO ihre Projekte nicht alleine aus dem Zustupf der Regierungen finanzieren und ist damit auf Drittmittel angewiesen. Im Gesundheitswesen spielt beispielsweise die Weltbank eine wichtige Rolle. Bei all diesen Projekten findet aber auch ein ungeheurer Wettbewerb zwischen den Industrien statt. 